\title{
Le $500^{\mathrm{e}}$ anniversaire de Léonard de Vinci, de la commémoration à la recherche : modèles et modélisation
}

Marie Thébaud-Sorger

\section{OpenEdition} Journals

Édition électronique

URL : http://journals.openedition.org/artefact/5922

DOI : 10.4000/artefact.5922

ISSN : 2606-9245

Éditeur :

Association Artefact. Techniques histoire et sciences humaines, Presses universitaires du Midi

Édition imprimée

Date de publication : 15 juillet 2020

Pagination : 259-262

ISBN : 978-2-8107-0691-4

ISSN : 2273-0753

Référence électronique

Marie Thébaud-Sorger, « Le 500e anniversaire de Léonard de Vinci, de la commémoration à

la recherche: modèles et modélisation », Artefact [En ligne], 12 | 2020, mis en ligne le 21 décembre 2020, consulté le 23 décembre 2020. URL : http://journals.openedition.org/artefact/5922 ; DOI : https://doi.org/10.4000/artefact.5922

\section{(9) $\odot \Theta \Theta$}

Artefact, Techniques, histoire et sciences humaines est mise à disposition selon les termes de la Licence Creative Commons Attribution - Pas d'Utilisation Commerciale - Pas de Modification 4.0 International. 


\section{Le $500^{\mathrm{e}}$ anniversaire de Léonard de Vinci, de la commémoration à la recherche : modèles et modélisation}

S i les commémorations constituent une opportunité pour attirer l'attention du grand public et les soutiens d'institutions diverses (culture, enseignement supérieur et recherche, etc.) elles sont aussi l'occasion de faire le point sur un état de la connaissance. Avec une figure aussi prestigieuse que Léonard de Vinci, l'année 2019 a marqué un temps fort, et a permis de mettre à jour l'historiographie passée au prisme de nouvelles approches. Ainsi les nombreuses expositions, conférences et publications auxquelles ce $500^{e}$ anniversaire a donné lieu, ont mis en lumière les multiples facettes de son activité - l'homme de l'art, l'ingénieur militaire et hydraulique aux côtés de la figure du peintre et de l'architecte - et remis en perspective la construction à différentes époques de la figure du " génie ». Comme l’a montré Pascal Brioist, les savoirs du maître toscan appartiennent autant à la synthèse des savoirs médiévaux qu’à la culture humaniste, nuançant son image à la fois solitaire et visionnaire' ${ }^{1}$. Les multiples événements organisés ont aussi permis de resituer et contextualiser sa production dans la pluralité de ses champs d'action. Certaines initiatives ont plus précisément fait la part belle aux sciences et aux techniques, à l'instar de l'exposition tenue à la British Library, Leonardo da Vinci: A Mind in Motion - et les articles du catalogue éponyme qui rassemble

1. Brioist, 2013 et 2019 ; Dubourg-Glatigny, 2016.

95 Marie Thébaud-Sorger, « Le $500^{\mathrm{e}}$ anniversaire de Léonard de Vinci, de la commémoration à la recherche : modèles et modélisation », Artefact, 12, 2020, p. 259-262. 
des contributions de spécialistes. Exposant les pages du précieux Codex, celle-ci retraçait la cohérence d'une pensée du mouvement animant toute une série de domaines (hydraulique, mécanique, astronomie et optique) sous-tendus par une même perception du monde naturel : il en appréhende les dynamiques, le rapport des forces, de résistances et de mouvements perpétuels, les relations formelles entre microcosme et macrocosme ${ }^{2}$.

D'autres expositions qui ont fleuri dans le sillage des activités commémoratives et que ce court dossier souhaite mettre en lumière - exposition permanente à Milan, temporaires à Romorantin et Oxford - furent l'occasion de tisser de nouvelles approches sur les techniques qui ne se restreignent pas à l'histoire mieux connue des machines, dispositifs hydrauliques et projets imaginaires (machines volantes et sous-marines), mais abordent aussi les correspondances structurelles dans la pensée technique et le rôle des modèles dans les processus de construction des savoirs.

Claudio Giorgione, curateur de la riche collection de modèles du Museo nazionale della scienza e della tecnologia "Leonardo da Vinci " ̀̀ Milan, revient sur la mise en place des nouvelles galeries ouvertes au musée en décembre 2019. Entreprendre l'itinéraire de ces modèles permet de resituer historiquement leurs usages selon leur contexte de production et de médiations spécifiques : fabriqués dans l'Italie de l'après-guerre, ils ont participé à la construction patrimoniale du génie léonardien pour les publics ${ }^{3}$. La muséographie joue un rôle central dans la recherche sur les savoirs techniques à travers l'organisation de ces galeries. Celles-ci ont pour fonction de contextualiser la production des savoirs de Léonard sur la nature et de transmettre les recherches les plus récentes par une scénographie contemporaine en construisant de nouvelles narrations qui mettent en scène et en perspective ces artefacts divers (modèles anciens, fac-similé, moulages, fresques, audiovisuels, etc.).

Les modèles de Léonard en trois dimensions ont été réalisés à partir d'esquisses et de dessins et n'étaient pas originellement destinés à être exécutés. Les productions des années cinquante sont des interprétations $s^{4}$, plus ou moins fidèles, par les contemporains engageant alors un dialogue entre passé et présent. Cette pratique a suscité également de nouvelles recherches

\footnotetext{
2. Barone, 2019.

3. Giorgione, 2019.

4. Giorgione, 2015.
} 
à l'instar du travail engagé par Andrea Bernadoni et Alexander Neuwhal. Ces derniers ont étudié et réalisé une série de modèles $3 \mathrm{D}$ à partir du codex Atlanticus (1585-1587) en modélisation virtuelles. Poursuivant leur investigation et leur collaboration avec Pascal Brioist, ils ont réalisé pour l'exposition de Romorantin, S'inspirer du vivant, des maquettes imitant rigoureusement les dessins et utilisant cette fois-ci les matériaux suggérés par Léonard. Il s'agissait de proposer au public d'explorer la relation entre figurations formelles et observations de la nature chez Léonard, en résonance avec les problématiques actuelles sur le biomimétisme. La scénographie de l'exposition permettait de réfléchir aux analogies de structures en mettant en correspondance panneaux, dessins, maquettes mais aussi machines contemporaines, source de créativité à la fois scientifique, mais aussi artistique.

La place de la modélisation dans les processus visuels, sensibles et tactiles de construction des savoirs était également au cour de l'exposition tenue à la Weston Library à Oxford, Thinking $3 D$ : Leonardo to the present, pilotée par Laura Moretti et Daniel Green. Liée à un plus large projet de recherche ${ }^{6}$, l'historienne de l'art et de l'architecture Katie Jacobiec, qui y a collaboré, retrace le dispositif de l'exposition. Il s'agissait également de présenter les différentes techniques utilisées au cours du temps (modélisation, photographie et stéréoscopie) pour réaliser des formalisations en trois dimensions dans des domaines tels que l'anatomie, l'architecture, l'astronomie et la géométrie. À partir des savoirs mathématiques mobilisés par Léonard puis dans les nombreux ouvrages diffusant la perspective et les nouvelles techniques de modélisation et de mesure, l'exposition mettait en évidence les relations entre les objets tridimensionnels et les formes visuelles et analytiques bidimensionnels. La tridimensionnalité incorporée dans les ouvrages et les modèles réalisés devient alors un vecteur de traduction des savoirs et de communication? ${ }^{7}$.

Ces expériences variées pour les visiteurs ouvrent des chemins fructueux sur l'apport de la culture visuelle et de la modélisation dans la transmission sensible et matérielle d'idées, de concepts et de processus techniques. Ainsi

\footnotetext{
5. Bernadoni et Neuwahl, 2014.

6. Voir le projet, https://www.thinking3d.ac.uk/about/ et la conférence internationale tenue à Worcester College les 27-28 septembre 2019, Thinking 3D Architecture and Audience.

7. Green, Moretti, 2019.
} 
tout en se destinant à des publics larges, ces expositions ont su mettre l'accent sur des pistes de recherches nouvelles et prometteuses.

\section{Marie Thébaud-Sorger \\ CNRS/Centre Alexandre-Koyré (UMR 8560)/MFO}

\section{Bibliographie}

BArone Juliana (dir.), Leonardo de Vinci: A mind in Motion, British Library, 2019.

Bernardoni Andrea, Neuwahl Alexander, Construire à la Renaissance : les engins de chantier de Léonard de Vinci, Presses universitaire de Rennes/Presses universitaires François Rabelais, Rennes, 2014.

Brioist Pascal, Les Audaces de Léonard de Vinci, Stock, Paris, 2019.

BRioist Pascal, Léonard de Vinci, homme de guerre, Alma éditeur, Paris, 2013.

Dubourg-Glatigny Pascal, "Pascal Brioist, Léonard de Vinci, homme de guerre ", Artefact. Techniques, histoire et sciences humaines, $\mathrm{n}^{\circ}$ 4, 2016, p. 411-413.

Green Daryl, Moretti Laura (dir.), Thinking 3D: Books, Images and Ideas from Leonardo to the Present, Bodleian Library, Oxford, 2019.

Giorgione Claudio, "The birth of a collection in Milan: from the Leonardo Exhibition of 1939 to the opening of the National Museum of Science and Technology in 1953 ", Science Museum Group Journal, n 4, 2015 [http:// dx.doi.org/10.15180/150404].

Giorgione Claudio, Leonardo Da Vinci. The Models Collection, Museo Nazionale della Scienza e della tecnologia Leonardo da Vinci, Museum collections, Milan, 2009. 